\title{
The quest for reliable biomarkers to identify new
}

\section{and recurrent cancer}

\begin{abstract}
"In this issue of Biomarkers in Medicine, we review the state of the science in biomarker development for breast, ovarian, prostate and pancreatic cancer."
\end{abstract}

There has been measurable progress in personalizing the treatment of adult malignancies. Once the diagnosis of cancer is made and the tumor is available, it can be genotyped and phenotyped to optimize the intervention that will be administered. Moreover, large-scale assessment of tumors has allowed the development of targeted therapies based on the mutations that drive tumor progression. Far less progress has been made in the development of biomarkers for the diagnosis of new or recurrent cancer. Perhaps this is not surprising, given the multitude of mutational and epigenetic events that can occur during cancer development and progression. In this issue of Biomarkers in Medicine, we review the state of the science in biomarker development for breast, ovarian, prostate and pancreatic cancer.

Tang and Gui review the current state of breast cancer biomarker research, and future directions [1]. They present four principal areas of promise from biomarker development: combining new with established markers to optimize cancer diagnosis; using biomarkers for the early determination of treatment response and disease recurrence/survival; guiding therapy; and facilitating the identification of the most promising drug candidates for therapeutics worthy of further development. The articles in this themed issue focus on the first and second areas: the diagnosis of new and recurrent cancer. The authors review several serum and tissue markers that have been identified as clinically useful in the management of patients with breast cancer. One major advantage of biomarker research for individuals with cancer is the presence of tumor tissue for analysis, which is not present in individuals without such a diagnosis. Tissue sampling in the absence of a suspicion of cancer is difficult to justify. For this reason, body fluid analysis holds greater promise in biomarker development for individuals without the presence of a lesion that requires biopsy. The authors rightly point out that currently approved biomarkers have focused on the assessment of single proteins, even though no single biomarker has demonstrated sufficient sensitivity and reproducibility for clinical use in the diagnosis of breast cancer among individuals presumed to be healthy and disease free. Approved biomarkers for the diagnosis of recurrent breast cancer perform rather poorly and are not in routine use in most large centers. The authors cite investigations with autoantibodies and DNA methylation as holding particular promise in biomarker development for the diagnosis of new and recurrent breast cancer.

For many years, prostate-specific antigen (PSA) was considered one of the best diagnostic cancer markers, and was part of clinical practice. However, the lack of specificity of PSA as a marker for prostate cancer, combined with the relatively benign nature of prostate cancer progression in many men who develop the disease has led clinicians to question its continued use. Because national bodies such as the US Preventive Services Task Force now recommend against PSA screening for prostate cancer, the research community has searched for other noninvasive markers such as proteins, circulating tumor cells and nucleic acids in the blood or urine of patients with prostate cancer. As Sardana and Diamandis point out, these markers, in combination with PSA, are being evaluated to develop a multiple-biomarker approach to the diagnosis of new and recurrent prostate cancer [2].

Adenocarcinoma of the pancreas (pancreatic cancer) is generally lethal. The reasons for this are many, but certainly one of the most important is late diagnosis. Survival is rare in the absence of early diagnosis, yet $>50 \%$ of individuals with very small lesions confined to the pancreas are alive 5 years after diagnosis. Indeed, as Batra et al. point out, pancreatic cancers on average have a relatively long time

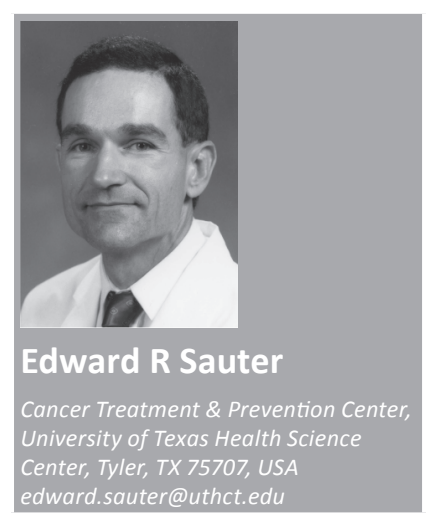

Future
Medicine 
(17 years) from tumor-initiating cell to tumor metastases, suggesting that it should be possible to find and treat the disease before it spreads [3]. Nonetheless, because of nonspecific symptoms and the lack of a good biomarker, overall 5-year survival remains a dismal $6 \%$ or less. Imaging is being investigated for the early detection of pancreatic cancer in high-risk individuals. Current limitations of imaging include high cost, limited sensitivity in detecting lesions $<1 \mathrm{~cm}$ in diameter and limited specificity, which can lead to invasive diagnostic interventions for lesions that are often found to be benign. Moreover, as the authors point out, biomarker sensitivity is reduced due to the asymptomatic nature of precursor lesions, the low level of secreted molecules in tissues and fluids and the lack of tissue and body fluid samples from individuals with early-stage disease.

"This issue of Biomarkers in Medicine outlines the state of the science, challenges and promising avenues for current and future research to identify better biomarkers."

There are no serologic markers for the early diagnosis of pancreatic cancer. CA19-9, the only marker in common clinical use to follow therapeutic response to pancreatic cancer treatment, has low sensitivity and specificity for the early detection of the disease. In addition to blood and blood products, investigators are evaluating pancreatic juice, stool and tumoral or peritumoral tissue. New avenues of investigation need to be explored, such as rodent models of early-stage disease and the validation of markers using samples collected from individuals with early-stage disease.

Survival from ovarian cancer is worse than other gynecologic malignancies, in part due to delay in diagnosis. The most commonly used biomarker, CA125, is useful in monitoring individuals with the disease, but not in early diagnosis due to its lack of specificity as a single biomarker. On the other hand, as Leung, Kulasingam and Diamandis point out, there is increasing evidence that a panel of markers that includes CA125 may be useful in early ovarian cancer detection [4]. First, the Risk of Malignancy Index, which incorporates imaging, a clinical variable (menopausal status) and CA125 to predict malignant disease in women presenting with a pelvic mass, demonstrated respectable sensitivity and specificity rates. Two other biomarker panels that evaluate multiple serum biomarkers - ROMATM (CA125 and HE4) and OVA1 ${ }^{\mathrm{TM}}$ (CA125, $\beta$-2 microglobulin, transferrin, transthyretin and ApoA1) - have received US FDA approval for discriminating between the benign or malignant nature of pelvic masses. There is no FDA-approved biomarker(s) for the detection of ovarian cancer in individuals lacking a pelvic mass.

In summary, there is a significant effort to identify sensitive and specific biomarkers for the early detection of new and recurrent cancer. This issue of Biomarkers in Medicine outlines the state of the science, challenges and promising avenues for current and future research to identify better biomarkers. The development of highly predictive biomarkers for early cancer detection remains a key approach to improving survival in each of these cancers, in which the sequelae of late-stage disease, which is generally not curable, are the reason for death.

\section{Financial \& competing interests disclosure}

The author has no relevant affiliations or financial involvement with any organization or entity with a financial interest in or financial conflict with the subject matter or materials discussed in the manuscript. This includes employment, consultancies, honoraria, stock ownership or options, expert testimony, grants or patents received or pending, or royalties.

No writing assistance was utilized in the production of this manuscript.

\section{References}

1 Tang SSK, Gui GPH. Biomarkers in the diagnosis of primary and recurrent breast cancer. Biomarkers Med. 6(5), 567-585 (2012).

2 Sardana G, Diamandis EP. Biomarkers for the diagnosis of new and recurrent prostate cancer. Biomarkers Med. 6(5), 587-596 (2012).

3 Kaur S, Baine MJ, Jain M, Sasson AR, Batra SK. Early diagnosis of pancreatic cancer: challenges and new developments. Biomarkers Med. 6(5), 597-612 (2012).
4 Leung F, Diamandis EP, Kulasingam V. From bench to bedside: discovery of ovarian cancer biomarkers using highthroughput technologies in the past decade. Biomarkers Med. 6(5), 613-625 (2012). 